\title{
Application of Hybrid Models to Blood Cell Production in the Bone Marrow
}

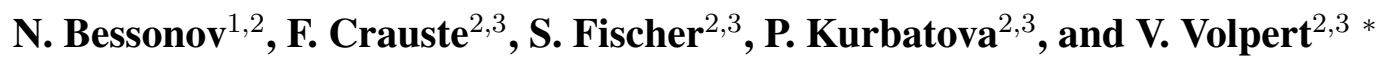 \\ ${ }^{1}$ Institute of Mechanical Engineering Problems, 199178 Saint Petersburg, Russia \\ ${ }^{2}$ Institut Camille Jordan, Université Lyon 1, UMR 5208 CNRS \\ 69622 Villeurbanne, France \\ 3 INRIA Rhône-Alpes, Team-project "Dracula"
}

\begin{abstract}
A hybrid model of red blood cell production, where cells are considered as discrete objects while intra-cellular proteins and extra-cellular biochemical substances are described with continuous models, is proposed. Spatial organization and regulation of red blood cell production (erythropoiesis) are investigated. Normal erythropoiesis is simulated in two dimensions, and the influence on the output of the model of some parameters involved in cell fate (differentiation, self-renewal, and death by apoptosis) is studied.
\end{abstract}

Key words: hybrid models, erythropoiesis, regulatory mechanisms

AMS subject classification: $92 \mathrm{C} 37$

\section{Biology and modelling of hematopoiesis}

Blood cells are produced in the bone marrow (and the spleen in mice) from stem cells that are pluripotent cells with the ability to differentiate in cells of every blood lineage. The erythroid lineage of hematopoiesis starts with erythroid progenitors (immature cells) and results in production of red blood cells. Progenitors can self-renew (produce cells with the same maturity as the mother cell), differentiate or die by apoptosis. Differentiation of progenitor cells results in appearance of more and more mature cells, among which reticulocytes and erythrocytes represent the latest stages of erythroid differentiation. Almost mature cells leave the bone marrow and enter the blood flow where they become mature erythrocytes.

\footnotetext{
${ }^{*}$ Corresponding author. E-mail: volpert@math.univ-lyon1.fr
} 
Erythropoiesis is strongly controlled by several regulatory mechanisms. The role of erythropoietin (EPO), a glucoprotein produced by the kidneys, has been identified at the end of the 1980's, by Koury and Bondurant [16]. EPO is released in the bloodstream in response to a lack of circulating red blood cells and acts on erythroid progenitors in the bone marrow, by inhibiting their apoptosis, consequently allowing a larger production of erythroid cells. Other hormones (glucocorticoids for instance [14]) control erythropoiesis by acting on immature cell self-renewal, differentiation or apoptosis, at various stages of erythroid differentiation. Such controls are usually mediated by the number of circulating red blood cells (mature cells). A second type of regulatory mechanisms acts spatially, in the bone marrow. Reticulocytes produce Fas-ligand which is fixed to their exterior cell membrane. Fas-ligand activates intracellular protein Fas in erythroid progenitors and influence their differentiation and apoptosis [17]. Erythroid cells can then interact in the bone marrow with each other and with the surrounding medium mechanically and biochemically.

In this work, we propose a new erythropoiesis model describing both intra-cellular regulatory networks and extra-cellular bio-chemical spatial regulation. We will study cell population dynamics with so-called hybrid models where cells are considered as discrete or individual based objects while intra-cellular and extra-cellular biochemical substances are described with continuous models. Erythroid cell behavior will then be determined by ordinary differential equations describing intra-cellular regulation, and by partial differential equations describing extra-cellular regulation. Hybrid models can be based on cellular automata or various lattice or off-lattice approaches (see $[1,2,3,13]$ and the references therein).

Previous attempts to model erythropoiesis focused mainly on continuous models, described by partial differential equations or delay differential equations $[4,10,11,12]$. These models successfully described stress erythropoiesis (anemia), yet they did not discuss normal erythropoiesis. Moreover, they only concentrated on the role and relevance of feedback controls in cell differentiation, and did not consider spatial aspects of erythropoiesis. Inspired by these works, we propose a different approach, considering both continuous regulation of erythropoiesis through feedback controls and spatial organization, via discrete models.

The next section is devoted to the presentation of our hybrid modeling, with examples in one dimension. Section 3 focuses on the core of this work, a two-dimensional model of erythropoiesis with results on normal erythropoiesis modeling.

\section{Hybrid models}

\subsection{General framework}

Let consider a framework in which cells can interact with each other and with the surrounding medium mechanically and biochemically, they can also divide, differentiate and die due to apoptosis. Hereafter we describe our approach of mechanical interactions between cells, then we focus on intra-cellular regulation, described by ordinary differential equations, and on extra-cellular regulation, described by partial differential equations.

In order to describe mechanical interaction between cells, we restrict ourselves here to the simplest model where cells are represented as elastic balls. Consider two elastic balls with the 
centers at the points $x_{1}$ and $x_{2}$ and with the radii, respectively, $r_{1}$ and $r_{2}$. If the distance $d_{12}$ between the centers is less than the sum of the radii, $r_{1}+r_{2}$, then there is a repulsive force between them, denoted by $f_{12}$, which depends on the distance $d_{12}$. If a particle with the center at $x_{i}$ is surrounded by several other particles with the centers at the points $x_{j}, j=1, \ldots, k$, then we consider the pairwise forces $f_{i j}$ assuming that they are independent of each other. This assumption corresponds to small deformation of the particles. Hence, we find the total force $F_{i}$ acting on the $i$-th particle from all other particles, $F_{i}=\sum_{j \neq i} f_{i j}$. The motion of the particles can now be described as the motion of their centers. By Newton's second law

$$
m \ddot{x}_{i}+\mu m \dot{x}_{i}-\sum_{j \neq i} f\left(d_{i j}\right)=0,
$$

where $m$ is the mass of the particle, the second term in the left-hand side describes the friction by the surrounding medium. Dissipative forces can also be written in a different form. This is related to dissipative particle dynamics [15].

Intra-cellular regulatory networks for the $i$-th cell are described by a system of ordinary differential equations

$$
\frac{d u_{i}}{d t}=F\left(u_{i}, u_{e}\right)
$$

where $u_{i}$ is a vector of intra-cellular concentrations, $u_{e}$ is a vector of extra-cellular concentrations, $F$ is the vector of reaction rates which should be specified for each particular application. Evolution of the concentrations of the species in the extra-cellular matrix is described by the diffusion equation

$$
\frac{\partial u_{e}}{\partial t}=D \Delta u_{e}+G\left(u_{e}, c\right)
$$

where $c$ is the local cell density, $G$ is the rate of consumption or production of these substances by cells. These species can be either nutrients coming from outside and consumed by cells or some other bio-chemical products consumed or produced by cells. In particular, these can be hormones or other signaling molecules that can influence intra-cellular regulatory networks. In some cases, convective motion of the medium should be taken into account. We do not discuss here various details of this model related to cell division, the force $f_{i j}$ and cell displacement, relation between discrete and continuous models of cell population, more complex cell geometry, influence of stochasticity and so on (see [5]-[8] for more details).

\subsection{D model examples}

We begin with the 1D model example where cells can move along the line. The coordinates $x_{i}$ in equation (2.1) are real numbers. Each cell can divide or die by apoptosis. After division a cell gives two cells identical to itself (this is self-renewal, there is no differentiation). We suppose that cell division and death are influenced by some bio-chemical substances produced by the cells themselves. 
We consider the case where there are two such substances, whose concentrations are denoted by $u_{e}$ and $v_{e}$ and satisfy the following system of equations:

$$
\left\{\begin{aligned}
\frac{d u_{e}}{d t} & =d_{1} \frac{\partial^{2} u_{e}}{\partial x^{2}}+b_{1} c-q_{1} u_{e} \\
\frac{d v_{e}}{d t} & =d_{2} \frac{\partial^{2} v_{e}}{\partial x^{2}}+b_{2} c-q_{2} v_{e}
\end{aligned}\right.
$$

These equations describe the evolution of the extracellular concentrations $u_{e}$ and $v_{e}$ with their diffusion, production terms proportional to the concentration of cells $c$ and with the degradation terms. We note that cells are considered here as point sources with a given rate of production of $u$ and $v$. The cell concentration is understood as a number of such sources in a unit volume. In numerical simulations, where cells have a finite size, we consider them as distributed sources and specify the production rate for each node of the numerical mesh.

Intra-cellular concentrations $u_{i}$ and $v_{i}$ in the $i$-th cell are described by the equations:

$$
\left\{\begin{aligned}
\frac{d u_{i}}{d t} & =k_{1}^{(1)} u_{e}(x, t)-k_{2}^{(1)} u_{i}(t)+H_{1}, \\
\frac{d v_{i}}{d t} & =k_{1}^{(2)} v_{e}(x, t)-k_{2}^{(2)} v_{i}(t)+H_{2} .
\end{aligned}\right.
$$

Here and in what follows we write equations for intra-cellular concentrations neglecting the change of the cell volume. This approximation is justified since the volume changes only twice before cell division and this change is relatively slow. The first term in the right-hand side of the first equation shows that the intra-cellular concentration $u_{i}$ grows proportionally to the value of the extra-cellular concentration $u_{e}(x, t)$ at the space point $x$ where the cell is located. It is similar for the second equation. These equations contain degradation terms and constant production terms, $H_{1}$ and $H_{2}$. When a new cell appears, concentrations $u_{i}$ and $v_{i}$ are set to zero.

If the concentration $u_{i}$ attains some critical value $u_{c}$, then the cell divides. If $v_{i}$ reaches $v_{c}$, the cell dies. Consider first the case where $k_{1}^{(1)}=k_{1}^{(2)}=k_{2}^{(1)}=k_{2}^{(1)}=0$. Then $u_{i}$ and $v_{i}$ are linear functions of time which reach their critical values at some times $t=\tau_{u}$ and $t=\tau_{v}$, respectively. If $\tau_{u}<\tau_{v}$, then all cells will divide with a given frequency, if the inequality is opposite, then all cells will die.

Next, consider the case where $k_{1}^{(1)}$ is different from zero (see [8] for other examples). If it is positive, then cells stimulate proliferation of the surrounding cells, if it is negative, they suppress it. Both cases can be observed experimentally. We restrict ourselves here by the example of negative $k_{1}^{(1)}$. All other coefficients remain zero. Therefore, cells have a fixed life time $\tau_{v}$. If they do not divide during this time, they die.

We carry out the 1D simulation where cells can move along the straight line. Initially, there are two cells in the middle of the interval. Figure 1 shows the evolution of this population in time. For each moment of time (vertical axis) we have the positions of cells (horizontal axis) indicated with blue points.

The evolution of the cell population in Figure 1 (left) can be characterized by two main properties. First of all, it expands to the left and to the right with approximately constant speed. Second, the total population consists of relatively small sub-populations. Each of them starts from 

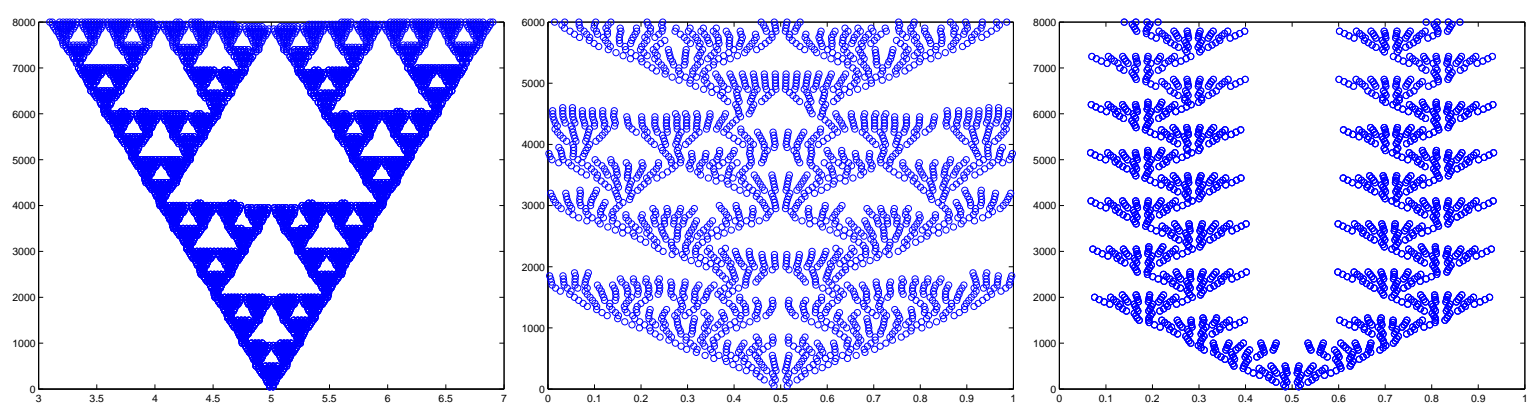

Figure 1: Dynamics of cell population in the case where cells either self-renew or die by apoptosis. Cells are shown with blue dots. Horizontal axis shows cell position, vertical axis shows time.

a small number of cells. Usually, these are two cells at the right and at the left of the previous sub-population. During some time, the sub-population grows, reaches certain size and disappears giving birth to new sub-populations.

This behavior can be explained as follows. The characteristic time of cell division is less than the one of cell death. When the sub-population is small, the quantity of $u_{e}$ is also small, and its influence on cell division is not significant. When the sub-population becomes larger, cell division is slowed down because of growth of $u_{e}$. As a result the sub-population disappears. The outer cells can survive because the level of $u_{e}$ there is less.

The geometrical pattern of cell distribution for these values of parameters reminds Serpinsky carpet (Figure 1, left), an example of fractal sets. The pattern of cell distribution depends on the parameters. Other examples are shown in Figure 1 (middle and right).

The simulations presented here do not use the extra-cellular variable $v_{e}$. Instead of the variable $u_{e}$, which decelerates cell proliferation, we can consider $v_{e}$ assuming that it accelerates cell apoptosis. In this case, qualitative behavior of cell population is similar.

\section{Erythropoiesis modeling}

\subsection{D hybrid model of erythropoiesis}

We consider two types of erythroid cells, progenitors and reticulocytes. Erythroid progenitor fate (differentiation, self-renewal, death by apoptosis) is supposed to be regulated by intra-cellular mechanisms (protein competition) and extra-cellular substances. The main external source of control in what follows will be Fas-ligand, a membrane protein produced by reticulocytes that activates the intra-cellular protein Fas. Other extra-cellular substances include EPO and glucocorticoids, among others. We will restrict our model to the influence of EPO, and even though we do not detail this action in the following, the level of EPO can be considered either constant or proportional to mature erythrocyte quantity. Fas-ligand will act on progenitor differentiation and apoptosis, whereas EPO will inhibit progenitor apoptosis and increase self-renewal.

We assume that intracellular regulation of erythroid progenitors is determined by two proteins, ERK and Fas (see [10]), although several other proteins may play a role in this regulation. A 
simplified model is given by the system of two ordinary differential equations [10]:

$$
\begin{gathered}
\frac{d E}{d t}=\left(\alpha(E P O)+\beta E^{k}\right)(1-E)-a E-b E F, \\
\frac{d F}{d t}=\gamma\left(F_{L}\right)(1-F)-c E F-d F,
\end{gathered}
$$

where $E$ and $F$ are intra-cellular concentrations of ERK and Fas, $a, b, c, d$ are some non-negative parameters, $\alpha$ is a function of erythropoietin (EPO) and $\gamma$ is a function of Fas-ligand, whose concentration is denoted by $F_{L}$. For fixed values of $E P O$ and $F_{L},(3.1)-(3.2)$ is a closed system of ordinary differential equations. It can have from one to three stationary points. Its detailed analysis is presented in [10].

The concentration of Fas-ligand is described by the diffusion equation

$$
\frac{\partial F_{L}}{\partial t}=d \Delta F_{L}+W-\sigma F_{L}
$$

where $W$ is a source term proportional to the concentration of reticulocytes. Though Fas-ligand is considered as a surface protein, and its interaction with erythroid progenitor basically occurs when they are in physical contact with reticulocytes, we model it as if it could diffuse in the extracellular matrix. If the diffusion coefficient is sufficiently small, it is located in a small vicinity of reticulocytes. Therefore, Fas-ligand influences erythroid progenitors when they are sufficiently close to reticulocytes.

Let us summarize the model. System (3.1)-(3.2) is considered inside each erythroid progenitor with its proper initial condition (see below) and with the value of $F_{L}$ which can depend on its spatial location and on time. Erythroid progenitors can proliferate or die by apoptosis. Apoptosis occurs if the intracellular Fas concentration reaches some critical value $F_{c}$. In this case, the cell is removed from the computational domain.

If the cell does not die by apoptosis, then it proliferates, that is it divides at the end of cell cycle. Cell cycle is composed of two parts: G0/G1 phases and S/G2/M phases. The duration of the G0/G1 phase is chosen randomly from 0 to some maximal value $\tau_{\max }$ with the typical values $6-12$ hours, the duration of the remaining part of cell cycle is fixed, usually 12 hours.

Cell proliferation can result in self-renewal or differentiation. In the first case, the two daughter cells are also erythroid progenitors. For each of them we consider intracellular regulation with system (3.1)-(3.2). The values of ERK and Fas in the newly born cells can either be some given parameters or equal half those of the mother cell. In the case of differentiation, the two daughter cells become reticulocytes. The choice between self-renewal and differentiation is determined by the values of ERK in the process of cell cycle. Once it reaches a critical value $E_{c}$, the cell selfrenews. Otherwise, it differentiates. These assumptions are in agreement with actual biological understanding of these processes.

It is noticeable that we do not consider intracellular regulation for reticulocytes. Once they appeared, they remain in the computational domain one cell cycle more in order to become mature erythrocytes. Then they are removed. This corresponds to the fact that erythrocytes leave the bone marrow to enter blood flow. 
Reticulocytes produce Fas-ligand with a constant rate. Fas-ligand influences intracellular regulation of erythroid progenitors through equation (3.2). It increases Fas production rate resulting in apoptosis of the progenitors if Fas concentration is sufficiently high or in their differentiation for intermediate values of $F_{L}$. For greater values of Fas-ligand, trajectories of system (3.1)-(3.2) move towards greater values of $F$ and to smaller values of $E$. Hence, the critical value of $E R K$ may not be reached and the cell will differentiate.

Let us finally recall that proliferation and apoptosis change cell spatial distribution. New cells, when they appear, push each other creating cell displacement. The equations of motion are described in Section 2.1.

\subsection{Erythroblastic islands}

We now study red blood cell production taking into account spatial cell organization that has been observed in the bone marrow but whose role in erythropoiesis, even though it seems quite central, is still largely unknown: erythroblastic islands (see for example [9]). In this structure, developing red blood cells surround a macrophage (white blood cell), immature cells being rather near the center of the island and mature cells slowly moving away from the macrophage in order to reach blood circulation.

For the simulations, as already mentioned, we only keep two sub-populations: immature progenitors, whose fate is described by system (3.1)-(3.2), at the center of the space and mature reticulocytes, secreting Fas-ligand, surrounding them. For simplicity, the macrophage itself has been omitted in these simulations (Figure 2). A more complete model of erythroblastic islands with central macrophages will be studied in subsequent works.

When simulating the model, in a wide range of parameters the structure of the island is conserved. The Fas-ligand gradient produced by reticulocytes induces apoptosis of the erythroid progenitors at the border of the island (high Fas-ligand and thus Fas values), differentiation for progenitors at intermediate distance (low Fas-ligand values) and self-renewal of the progenitors at the center of the island (no Fas-ligand). In this way, reticulocytes leaving the bone marrow are constantly replaced and there is always a belt of mature cells surrounding immature cells.

However, even if the topology of the island is conserved, depending on parameters, it may very well increase (excessive proliferation), decrease (extinction) or remain roughly at the same size (Figure 2). As random events occur (direction of division, length of cell cycle), small variations in the size of the island are observed in any case. It is interesting to study the impact of the main parameters on the overall behavior of the island and on the rate of production of mature red blood cells, which is the main physiological role of erythropoiesis.

The parameters of the main interest are those controlled by the extracellular regulation, namely the critical value $F_{c}$ of Fas for which apoptosis occurs and which is controlled by the hormone erythropoietin (EPO), and the parameter $\alpha$ in equation (3.1), which is also controlled by EPO as well as by some other hormones (glucocorticoids). Once the values of the other parameters are set, we can study their impact by seeing how the proportion of apoptotic, differentiating and selfrenewing progenitors vary. Figure 3 shows these proportions as a function of each parameter, the others remaining constant. 

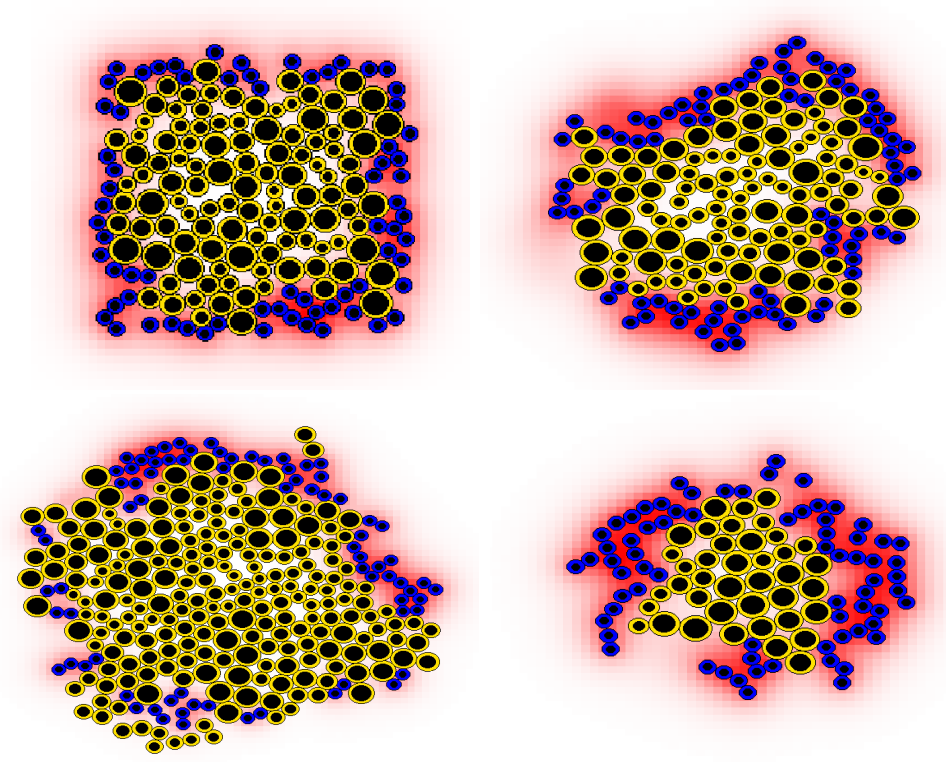

Figure 2: Initial cell distribution (upper left) and cell distributions after some time for three different cases: stable erythroblastic island with an approximately constant size (upper right), growing island (bottom left) and decreasing island (bottom right). Yellow cells in the center are immature cells, blue cells at the border are mature cells (reticulocytes) producing FasL (in red).
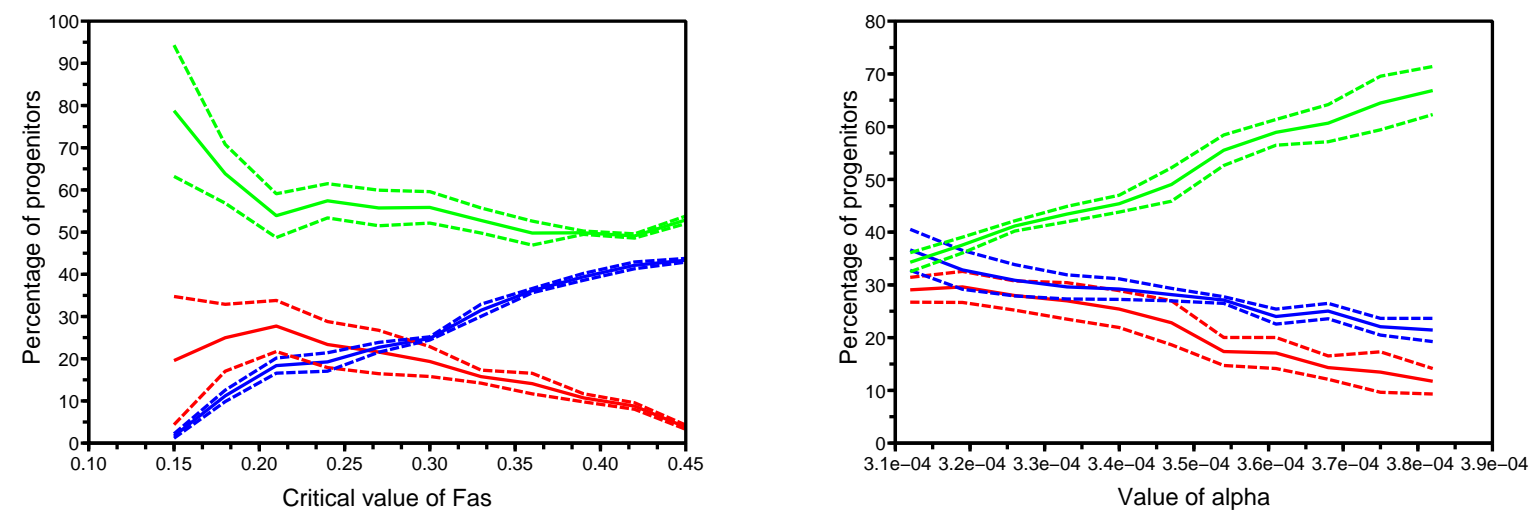

Figure 3: Proportion of apoptotic (red), differentiating (blue) and self-renewing (green) cells depending on the critical value of Fas (left) and on $\alpha$ (right). Thick lines indicate means and dotted lines standard deviation over 40 simulations.

As can be expected biologically, decreasing the critical value of Fas (which corresponds to an increase of EPO), clearly decreases apoptosis in favour of differentiation. The proportion of self-renewing cells remains approximately constant for the critical value of Fas greater than 0.2. Increasing $\alpha$ leads to more self-renewal, which is the observed effect of glucocorticoids [17]. The 
part of apoptotic and differentiating cells decreases while their ratio does not change.

Using these two parameters, it is thus possible to control the fate of the island: increasing $\alpha$ or decreasing the critical value of Fas will lead to its growth and reverse trends will lead to extinction. Since growth of the erythroblastic island implies production of more mature cells, EPO and glucocorticoids simulate the expected behavior of stress factors, which are expressed when erythrocytes in blood circulation are lacking.

Another interesting fact is that, even for intermediate values of $\alpha$ and of the critical value of Fas, it seems impossible to achieve complete stabilization of the island for the spatial structure defined above. After a while, the inherent small variations in size always lead to an unbalanced proportion of self-renewing and differentiating cells and thus either to unlimited growth or to extinction. However, the island remains stable during at least ten cycles. Therefore, this does not matter so much since, biologically, islands are rather expected to decrease, knowing that their full extinction might be prevented by the arrival of new progenitors produced by hematopoietic stem cells.

Simulating feedback through EPO or glucocorticoids does not really help improving the stability of the island: even though it has a clear impact on the speed of proliferation or extinction, it is not efficient enough to counter them rapidly. This is also understandable: Epo and glucocorticoids act at a global scale in response to global factors and do not aim at stabilizing local structures. It is more likely that the stability of islands is controlled by local factors, for example produced by the central macrophage. Many such factors have already been identified.

Finally, we can see that introducing a specific local structure helps us understanding the need of local and global control of erythropoiesis and how cells react to each of the existing factors. Islands in our model are robust structures reacting to stress factors by modulating their size and thus correctly answering to global needs of the organism but we see that additional factors or elements are needed to control these local structures.

\section{Acknowledgements}

The authors are grateful to the members of the INRIA team "Dracula" for stimulating discussions. F. Crauste, P. Kurbatova and V. Volpert's work is supported by the ANR grant ANR-09-JCJC0100-01

\section{References}

[1] A. R.A. Anderson. A hybrid multiscale model of solid tumour growth and invasion: Evolution and the microenvironment. in Single-Cell-Based Models in Biology and Medicine (Ed. A.R.A. Anderson, M.A.J. Chaplain and K.A. Rejniak), Series Mathematics and Biosciences in Interaction, Springer, Birkhauser Basel, 2007, 3-28.

[2] A.R.A. Anderson, M. Chaplain, K.A. Rejniak. Single cell based models in biology and medicine, Mathematics and Biosciences in Interaction. Springer, Birkhauser Basel, 2007. 
[3] A. R. A. Anderson, K.A. Rejniak, P. Gerlee, V. Quaranta. Modelling of cancer growth, evolution and invasion: bridging scales and models. Math. Model. Nat. Phenom., 2(3) (2007), $1-29$.

[4] J. Bélair, M.C. Mackey, J.M. Mahaffy. Age-structured and two delay models for erythropoiesis. Math. Biosci., 128 (1995), 317-346.

[5] N. Bessonov, L. Pujo-Menjouet, V. Volpert. Cell modelling of hematopoiesis. Math. Model. Nat. Phenom., 1 (2006), No. 2, 81-103.

[6] N. Bessonov, I. Demin, L. Pujo-Menjouet, V. Volpert. A multi-agent model describing selfrenewal or differentiation effect of blood cell population. Mathematical and Computer Modelling, 49 (2009), 2116-2127.

[7] N. Bessonov, P. Kurbatova, V. Volpert. Particle dynamics modelling of cell populations. Prooceedings of the conference JANO, Mohamadia 2008, Math. Model. Nat. Phenom., 5 (2010), No. 7, 42-47.

[8] N. Bessonov, P. Kurbatova, V. Volpert. Dynamics of growing cell populations. CRM, preprint num. 931 for Mathematical Biology, February 2010.

[9] J.A. Chasis, N. Mohandas. Erythroblastic islands: niches for erythropoiesis. Blood, 112 (2008), pp. 470-478.

[10] F. Crauste, I. Demin, O. Gandrillon, V. Volpert. Mathematical study of feedback control roles and relevance in stress erythropoiesis. J. Theo. Biol., 263 (2010), 303-316.

[11] F. Crauste, L. Pujo-Menjouet, S. Génieys, C. Molina, O. Gandrillon. Adding self-renewal in committed erythroid progenitors improves the biological relevance of a mathematical model of erythropoiesis. J. Theor. Biol., 250 (2008), 322-338.

[12] I. Demin, F. Crauste, O. Gandrillon, V. Volpert. A multi-scale model of erythropoiesis, J. Biol. Dyn. 4 (2010), pp. 59-70.

[13] D. Drasdo. Center-based single-cell models: An approach to multi-cellular organization based on a conceptual analogy to colloidal particles. In: Single-Cell-Based Models in Biology and Medicine (Ed. A.R.A. Anderson, M.A.J. Chaplain and K.A. Rejniak), Series Mathematics and Biosciences in Interaction, Springer, Birkhauser Basel, 2007, 171-196.

[14] O. Gandrillon, U. Schmidt, H. Beug, J. Samarut. TGF-beta cooperates with TGF-alpha to induce the self-renewal of normal erythrocytic progenitors: evidence for an autocrine mechanism. EMBO J., 18 (1999), 2764-2781.

[15] M. Karttunen, I. Vattulainen, A.Lukkarinen. A novel methods in soft matter simulations, Springer, Berlin, 2004. 
[16] M.J. Koury, M.C. Bondurant. Erythropoietin retards DNA breakdown and prevents programmed death in erythroid progenitor cells, Science, 248 (1990), 378-381.

[17] C. Rubiolo, D. Piazzolla, K. Meissl, H. Beug, J.C. Huber, A. Kolbus, M. Baccarini. A balance between Raf-1 and Fas expression sets the pace of erythroid differentiation. Blood, 108 (2006), 152-159. 